CuPAUAM 22, 1995, pp. 9-20

\title{
AVANCE SOBRE LOS TRABAJOS DE EXCAVACIÓN REALIZADOS EN EL YACIMIENTO DE EL HABARIO (PENDES-CANTABRIA): PROCESOS TÉCNICOS
}

\author{
Elena CARRIÓN SANTAFE* \\ JAVIER BAENA PREYSLER* \\ CARMEN CONDE RUIZ* \\ * Departamento Prehistoria y Arqueología \\ de la Universidad Autónoma de Madrid
}

\section{Resurnen}

En este trabajo son presentados algunos de los resultados prelininares de los testimonios encontrados en el yacimiento de El Habario, Jocalizado en las estribaciones orientales de los Picos de Europa a 536 metros s.n.m. Los hallazgos recuperados consisten fundamentalmente en nódulos y restos irabajados en cuarcita de origen local. El intenso estudio de atributos técnicos nos ha permitido obtener una secuencia de reducción que muestra un dominio en la explotación y producción de jascas con dos caras bulbares, junto a la ausencia relativa de materiales levallois. Todo ello nos permite dar una clasificacion provisional al yacimiento de "taller", encuadmble en fases recientes del Palcolítico Antiguo. Futuras investigaciones tratarán de estableccr la relación que este yacimiento guarda con posibles asentamientos en cleva cercanos.

\begin{abstract}
In this paper some preliminary aspects of the archcological remains found in the "El Habario" (PenciesCantabria) site are presented. This archeological site is located in the eastern part of the Picos de Europa Mountains, at 536 meters from the sea level. The remains recovered in this place are cobbles and lithic implements made in focal quarzcite, and apear in a mountain position in the open air. Through an exhatstive atribute analysis, the lithic reduction shows a clear sequence in which flakes with two bulbar faces (Kombewa) are the principal element, and no important levallois record are registred. This information alow us to clasificate the site provisionally as a "Middle" Paleolithic "Workshop site" Further investigations will analize the relation between this site and some other settlements.
\end{abstract}




\section{INTRODUCCIÓN}

El Habario es un yacimiento de superficie localizado en el término de Pendes-Castrocillorigo, en el sector occidental cántabro dentro de la comarca de la Liébana. La excavación de este yacimiento se integra dentro de un proyecto de investigación financiado por la Consejería de Cultura y Deporte de Cantabria, que con el título "Las estrategias de producción lítica durante el Paleolítico Inferior y Medio en el centro de la región cantábrica" trata de establecer cuáles han sido los principales modelos de actuación humana durante estos periodos en los distintos ambientes que la región presenta. Su localización en las estribaciones de Los Picos de Europa hace del yacimiento un lugar excepcional para tratar de conocer los procesos desarrollados por las comunidades recientes del $\mathrm{Pa}$ leolítico Antiguo en áreas internas de la región cantábrica.

Existen referencias previas de este yacimiento a través del los trabajos de CASTANEDo et al., 1993; estos autores realizan un estudio de las colecciones de superficie llegando a la conclusión de que el yacimiento es encuadrable dentro del Paleolítico Medio. Durante el año 1996, y dentro del proyecto antes mencionado, se emprendió una campaña de excavación en el mismo, en la que se realizaron distintos trabajos que pusieron de manifiesto la existencia de importantes acumulaciones de industria junto a cantos de cuarcita sin trabajar, que proceden de afloramientos de conglomerados ricos en cuarcitas armoricanas situados en las cercanías.

Los trabajos de excavación, así como el estudio posterior de los restos localizados nos han permitido establecer una secuencia de reducción que, con lagunas, va a ser un referente esencial en el conocimiento de la actividad de las primeras comunidades humanas que ocuparon la zona. Estos resultados, que se encuentran integrados en una trabajo de investigaciôn actualmente en curso (memoria de licenciatura titulada "El yacimiento de El Habario - Pendes, Cantabria - y su relación con las industrias musterienses al aire libre en el centro de la región cantábrican), pretenden determinar las posibles conexiones existentes entre yacimientos localizados en el entorno. Vamos a exponer a modo de avance, algunos de los resultados obtenidos en su estudio.

\section{Captación de materias primas (véase figura 1)}

La distancia del afloramiento original de cuarcitas respecto del depósito arqueológico es muy escasa (menos de $1 \mathrm{~km}$ ). No obstante no negamos la posibilidad de que la formación defina un espacio muy amplio susceptible de contener depósitos explotables por estas comunidades (en todo caso depósitos de carácter secundario).

El depósito arqueológico presenta una clara asociación estratigráfica entre bases naturales (cantos completos y fragmentos con forma de "tableta") y materiales trabajados. Todo guarda una relación de homogeneidad muy alta en lo referente a su grado de rodamiento/alteración. 
La presencia de estas tabletas en el depósito arqueológico puede ser explicada, además de por los procesos erosivos que formaron la acumulación, por la propia actividad de talla llevada a cabo sobre los depósitos de cantos de carácter secundario.

Se documenta igualmente la presencia, muy escasa por otra parte, de sílex de características locales. Hay que tener en cuenta sin embargo, que los afloramientos más cercanos de este material distan aproximadamente $2,5 \mathrm{~km}$. del yacimiento. Dicho sílex presenta una escasa aptitud para la talla, como apuntan algunos autores (ARIAS CABAL, 1987).

\section{Fases iniciales de explotación o tanteo}

Las estrategias de explotación llevadas a cabo en yacimiento han podido ser reconstruidas con éxito gracias a un examen detenido de los atributos técnicos y también, a la existencia de una fuerte homogeneidad en el mismo.

Los procesos documentados son los siguientes:

\section{A) Selección de matrices}

La selección de las matrices se realiza de dos formas distintas. Primero mediante la talla, tanto de cantos como de fragmentos, para la obtención de lascas. Además, dada la existencia de una alteración dominante en forma de tableta, se puede optar por la selección de estas matrices naturales. Hay que tener en cuenta que los modelos experimentales realizados sobre cantos de la zona muestran como la producción de estas tabletas se ve favorecida por la percusión. De esta forma la talla podría producir dos tipos de matrices mediante un mismo proceso: lascas y tabletas. El aprovechamiento como base de industria de fragmentos o planos de fractura naturales, fomentados o no por la talla, es bien conocido en numerosos yacimientos del Paleolítico Antiguo (figura 1).

En el primero de los casos, las lascas producidas guardan una relación dimensional bastante homogénea y son el resultado de una percusión directa, dlanzada? o apoyada en suelo, siempre realizada con percutores duros. En el segundo, dentro de los fragmentos aprovechados parece haber una tendencia al aprovechamiento de morfologias hemisféricas o en tableta, aunque este punto no ha podido comprobarse en un número suficiente de piezas.

Son muy escasos los testimonios en los que se recoge una explotación directa de los cantos. Siempre que ésta se produce, se realiza de forma poco intensa ( 2 o $3 \mathrm{ex}-$ tracciones como máximo); todo parece indicar que se tratan de golpes de tanteo. No deja de ser extraño el que sea esta primera fase de explotación la que esté peor representada. 


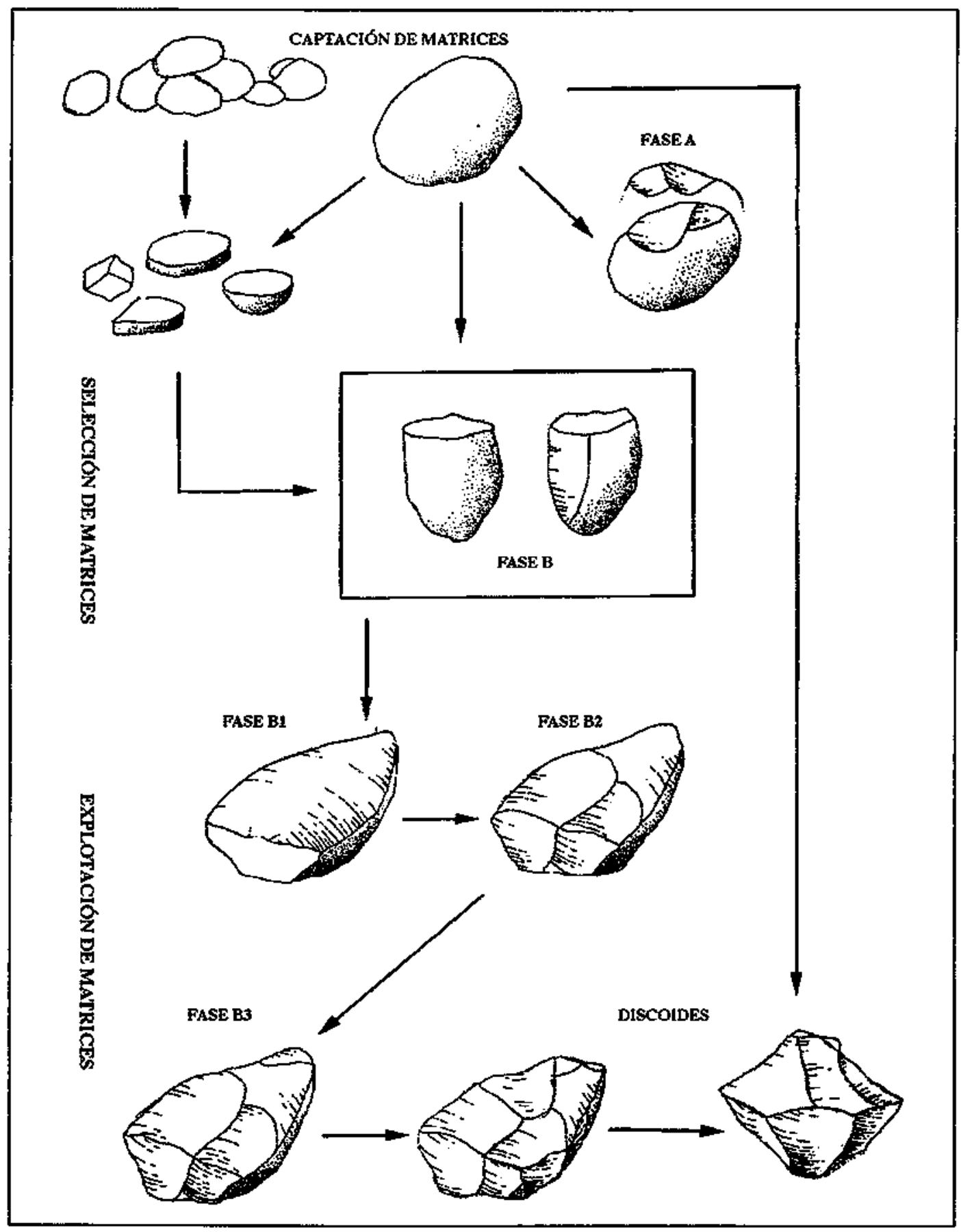

Figura 1 


\section{B) Producción inicial de lascas predeterminadas}

La estrategia principal documentada en el yacimiento consiste en el aprovechamiento de los planos de lascado de las matrices (lascas) obtenidas en la fase anterior (Figura 1. Fase B). El proceso de explotación muestra una gran homogeneidad en la mayoría de los núcleos del yacimiento, aunque los productos obtenidos presentan, aparentemente, un escaso grado de predeterminación.

La secuencia comienza en primer lugar con el reconocimiento de las distintas superficies que componen la lasca matriz; su talón, su reverso y su anverso. El primer paso comienza con la preparación de una futura superficie de percusión coincidiendo con la zona del talón (figura 1. Fase B). Una vez preparada, se procede a la explotación propiamente dicha, realizando las extracciones sobre el plano de lascado o reverso de la lasca matriz. Dentro de las estrategias de explotación, son frecuentes los núcleos que presentan una extracción a cada lado del cono de percusión (figura 1. Fase B). Como resultado de este proceso se obtienen numerosas lascas con doble plano de lascado (kombewas) (TXIER, 1980; SANTONJA, 1984-85, entre otros).

Los productos de esta fase (tanto los de preparación de plataformas, como las extracciones principales) son fácilmente discriminables (figura 2. A). Las extracciones de preparación presentan superficies corticales, con o sin planos negativos . Las extracciones principales se identifican por la presencia en el anverso, del antiguo plano de lascado de la lasca matriz, con o sin otros negativos de extracciones (figura 2 B). La caracterización de las primeras puede resultar compleja en ocasiones pues como dijimos, puede que se haya explotado planos producidos por fracturas naturales.

La significación de este tipo de proceso viene definida por la abundancia en el depósito, de núcleos que detienen su explotación en este punto y son abandonados. No obstante hemos de recordar que estamos hablando de un tipo de yacimiento muy particular en el que la asociación de materiales sin trabajar (mayoritarios) junto a mareriales trabajados está clara, pero en el que existen igualmente materiales que pueden asignarse a fases más avanzadas de esta secuencia (por ejemplo útiles retocados).

\section{C) Producción de lascas con baja predeterminacion (figura 1. Fase B3)}

Si continuamos con la explotación de los núcleos anteriores podremos generar núcleos en los que domina claramente una superficie de trabajo. La ausencia de alternancia è estos casos está clara. La continuidad en su explotación, con cierto grado de altemancia podría desembocar en la creción de núcleos discoidales. Lógicamente, no se ha podido establecer una conexión entre los tipos con una superficie de trabajo dominante y los discoidales.

En ambos casos, entramos dentro de una fase diferenciada en la cadena técnica llevada a cabo en el conjunto del Habario, y que consiste en el aprovechamiento centrípeto de los núcleos del tipo anterior. Para realizar esta fase de explotación se recurre principalmente al trabajo de forma bifacial no alternante de los mismos. 

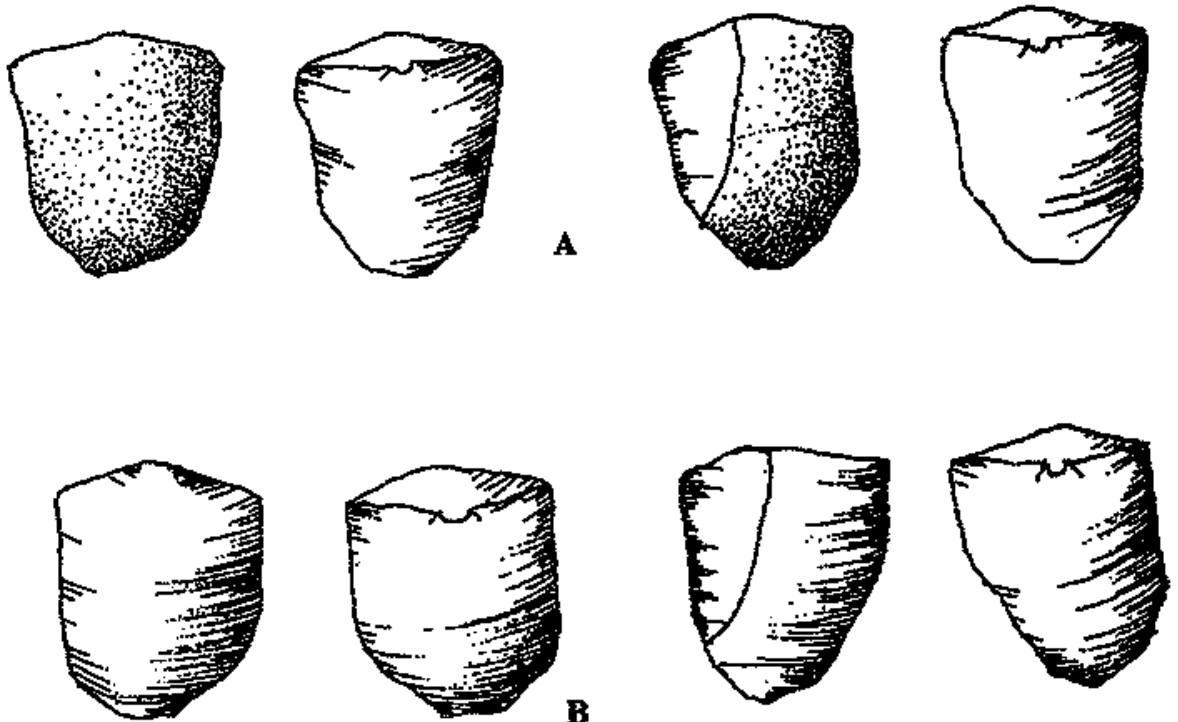

Figura 2

El proceso en su conjunto genera productos con un bajo número de extracciones en anverso junto a talones con uno o dos procesos de trabajo principalmente (lisos o diedros).

Su existencia se corresponde con núcleos más o menos discoidales en los que parece existir, como deciamos, una diferencia en el grado de trabajo de sus superficies (mayor grado de trabajo en las superficies principales, frente a menor en la superficie de preparación). Igualmente se observa una clara jerarquización de los hemisferios de los núcleos (volúmenes respecto al "plano intermedio"), tanto en los núcleos de esta fase como en los anteriores.

Este hecho parece significativo e indicador de grupos conocedores de la importancia de la posición relativa de las plataformas respecto al mencionado "plano intermedio", lo que puede ponerse en relación con el desarrollo temporal de la talla bifacial.

\section{D) Ausencia relativa de otros procesos técnicos}

Lo que más llama la atención, junto a los procesos descritos, es la ausencia de otros procesos técnicos. Este hecho confiere al conjunto una unidad técnica muy clara, y describe un proceso de captación-explotación inicial especializado, y en menor medida explotación intensa. 


\section{Secuencia y cronologia}

En la actualidad venimos estudiando la secuencia superior del yacimiento, en donde se pudo obtener mediante prospección y excavación un conjunto importante de materiales en un paquete estratigráfico con un grado muy alto de alteración (Habario A). Estos materiales, pueden corresponder a un periodo temporal muy amplio pero en cualquier caso se superponen estratigráficamente al conjunto de El Habario descrito (denominado Habario B).

El análisis preliminar de estos materiales ha puesto de manifiesto las siguientes caracteristicas:

a) Se trata de un conjunto que muestra modos de explotación sobre los mismos cantos de cuarcita. La industria presenta sin embargo un grado de alteración casi nulo.

b) Los modos de explotación son muy diversos, e incluyen trabajos de tanteo, explotaciones del tipo descrito en los niveles del Habario B, explotaciones paralelas sobre canto, trabajos con tendencia poliédrica, explotaciones centripetas sobre lasca, etc.

c) Destaca sin embargo la presencia de procesos de reducción recurrente centripeta levallois con presencia de algunos núcleos bastante clásicos.

d) Asociación de los núcleos con lascas y un material retocado de aspecto igualmente clásico.

La existencia en niveles cronológicamente posteriores (al menos asi lo hemos interpretado) de industrias musterienses "clásicas" puede ser un elemento de juicio impor" tanie para la evaluación cronológica del depósito de El Habario $\mathbf{B}$.

Desgraciadamente las condiciones edáficas no permiten la conservación de testiinonios que puedian ofrecer cronologias. Sin embargo, los rasgos técnicos hablan claramente, en ocasiones por ausencia de otros elementos, de un momento arcaico dentro del Paleolítico medio.

Contamos con algunos criterios como la secuencia climática (oscilación glaciar y su efecto local) o la relación con conjuntos cercanos, que nos permitirán precisar más la cronología del depósito.

\section{Consideraciones finales e interpretación del yacimiento}

Una vez expuesto el modelo de reducción, cabe plantearse una serie de reflexiones șobre la caracterización funcional del yacimiento.

Las posibilidades en este sentido pueden ordenarse de la siguiente forma: 
a) Empleo del lugar como área de captación o transformación de materia prima lítica.

b) Empleo del lugar como área de captación de recursos orgánicos.

c) Uso combinado del lugar (asentamiento/taller...).

Todo parecde indicar que el yacimiento puede encuadrarse en la categoría de taller. No obstante la escasa potencia del estrato que contiene los materiales, la ausencia de estructuras que señalen una ocupación reiterada, o el grado de homogeneidad presente en el conjunto, apunta a que no ha habido una ocupación prolongada del mismo. A falta de otros datos, podría asignarse al caso concreto de El Habario un escaso carácter estratégico.

Sin embargo las dificultades en la caracterización se multiplican si consideramos la posibilidad de que El Habario consista en una porción, poco significativa, de un conjunto de yacimientos extendidos por todo el entorno inmediato en función de la oferta litológica local. La segunda posibilidad es que nos encontremos ante un aprovechamiento "fortuito" e improvisado de un recurso no conocido previamente. Discernir entre ambos modelos sólo será posible con un conocimiento más exhaustivo del potencial arqueológico del entorno.

La categoría de los distintos elementos líticos que componen el yacimiento que estudiamos (El Habario B) apoyan la interpretación del mismo como taller:

- Núcleos: $11.9 \%$.

- Productos de lascado: $\mathbf{4 7 . 8 \%}$

- lascas de descorticado primario: $5.2 \%$,

- lascas de descorticado secundario: $15.6 \%$,

- lascas simples: $23.2 \%$,

- lascas Levallois: $2.5 \%$,

- láminas: $\mathbf{1 . 3 \%}$.

- Material retocado: $11.4 \%$.

- útiles: $11.4 \%$,

- lascas retocadas: $18.3 \%$.

- Restos de talla: $6.5 \%$.

- Debrises: $3.5 \%$.

La abundancia de material retocado en un yacimiento en principio clasificable como taller presenta problemas de interpretación. Varias son las posibilidades que explican estas proporciones de material retocado (relacionable con el consumo); 
- El material retocado fue llevado hasta el yacimiento por los grupos humanos que explotaron el mismo; su abandono posterior sugiere la utilización del utillaje en el lugar en relación con estrategias de aprovechamiento que desconocemos.

- El utillaje presente en el yacimiento supone tan sólo un parte del material que se retocó. Del total se seleccionó una parte para ser transportada y aprovechada en otros lugares, abandonándose en $\mathrm{El} \mathrm{Habario} \mathrm{los} \mathrm{materiales} \mathrm{no} \mathrm{deseados.}$

- Los materiales retocados representan actividades de aprendizaje o intercambio de información práctica en el grupo, por lo que las proporciones de tipos que antes apuntamos pueden entenderse como el resultado de "ensayos" sin utilidad directa.

En cualquier caso, hemos de contar con el hecho de que la presencia de material foráneo es prácticamente nula, salvo las escasas muestras de silex negro citadas anteriormenie. Todo parece apuntar que el material retocado (que guarda una gran coherencia iécnica con el resto de los materiales que componen el conjunto) fue fabricado en el propio yacimiento, o al menos se integra claramente con la secuencia de reducción.

Sin duda las actuaciones en las cuevas del entorno (lugares de hábitat potencial) son fundamentales para el conocimiento de las estrategias de aprovechamiento de estos gnupos. ¿Condiciona la necesidad de materia la presencia humana en aquellos parajes? $O$, por el contrario, ¿es el aprovechamiento de recursos locales no líticos lo que propicia el poblamiento y determina la explotación de la cuarcita local?

A falta de datos más ajustados sobre la cronología y paleoecología que acompañaron la actividad humana en la zona, consideramos probable una bonanza ambiental para estos momentos. Ello explica por una parte la ocupación de cotas tan hostiles en momentos de frí riguroso, tanto como la combinación de estrategias aire libre/cueva, que venimos proponiendo.

Futuras investigaciones deberán profundizar en el conocimiento del Paleolítico Medio local, hasta ahora poco conocido. Sumamente interesante nos parece la excavación de las cuevas de la comarca, que permitirá conectar las fases de las posibles cadenas operativas presentes en ellas con la funcionalidad y naturaleza presupuesta para $\mathrm{El} \mathrm{Ha-}$ bario. Con ello intentaremos dotar a El Habario, y a los hipotéticos refugios que se determinen, de un significado crono-cultural más ajustado, en el que se concrete la relación de estas zonas de producción con los materiales de yacimientos cercanos (Cueva de $\mathrm{Ca}$ bañes) o situados en áreas distantes (Cueva del Esquilleu; MuÑoz et al., 1985).

Así mismo se pretende investigar sobre la naturaleza de los yacimientos en cueva, para comprobar si la presencia humana en los mismos (todavía a constatar) responde a ocupaciones ocasionales o a una intencionalidad de hábitat prolongado. Igualmente se abordará la interpretación de la funcionalidad de las piezas tanto como la asociación con restos de otra naturaleza (faunísticos).

Esperamos que ello permita la confección de modelos aproximados sobre las estrategias de aprovechamiento del medio durante la última parte del Paleolítico Antiguo, así como determinar el cambio que éstas experimenten a través del tiempo. 


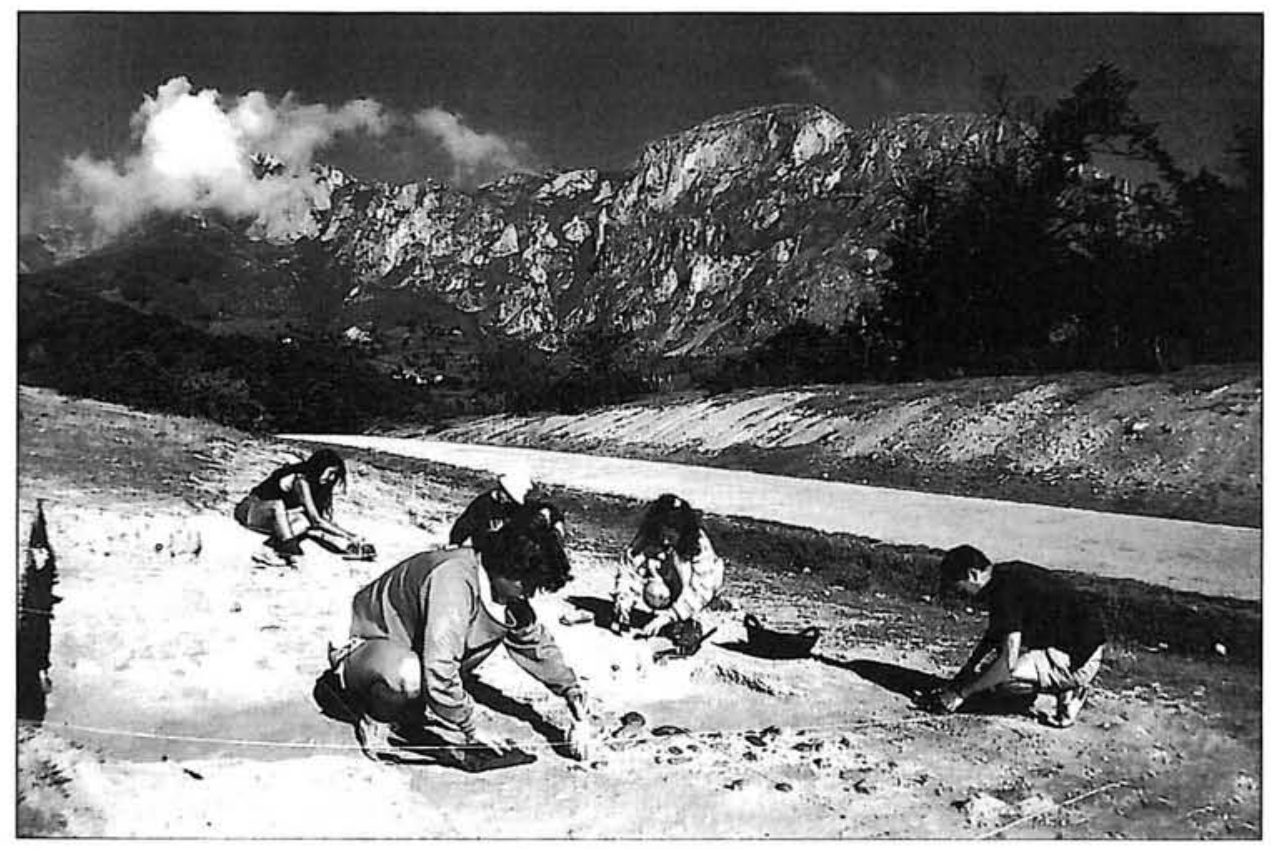

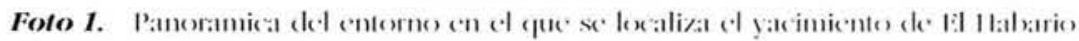

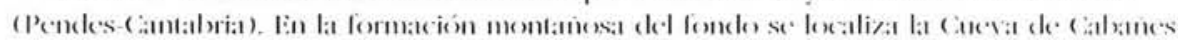

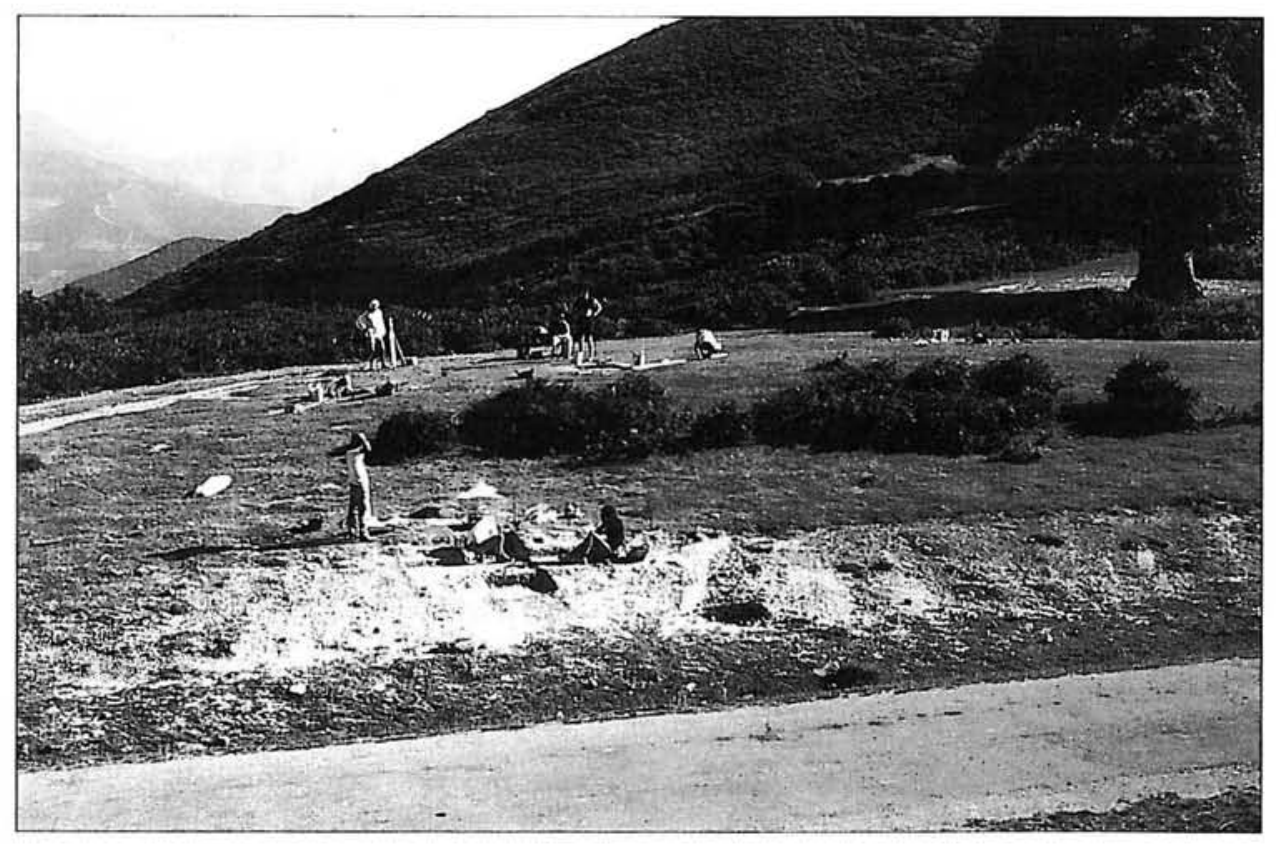

Foto 2. Vistit general del yacimicnte durante su fase de excasateion 


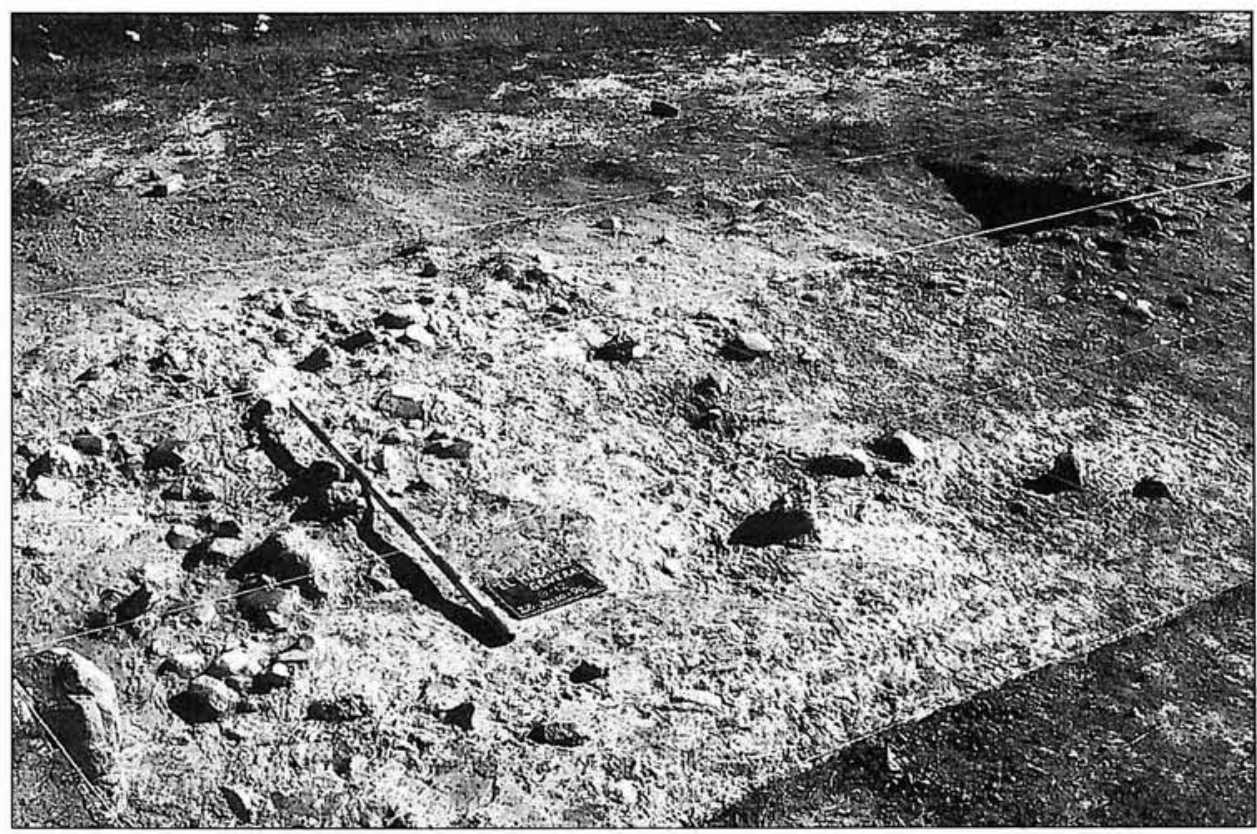

Foto 3. Nivel de excavación del yacimiento de lal I labario

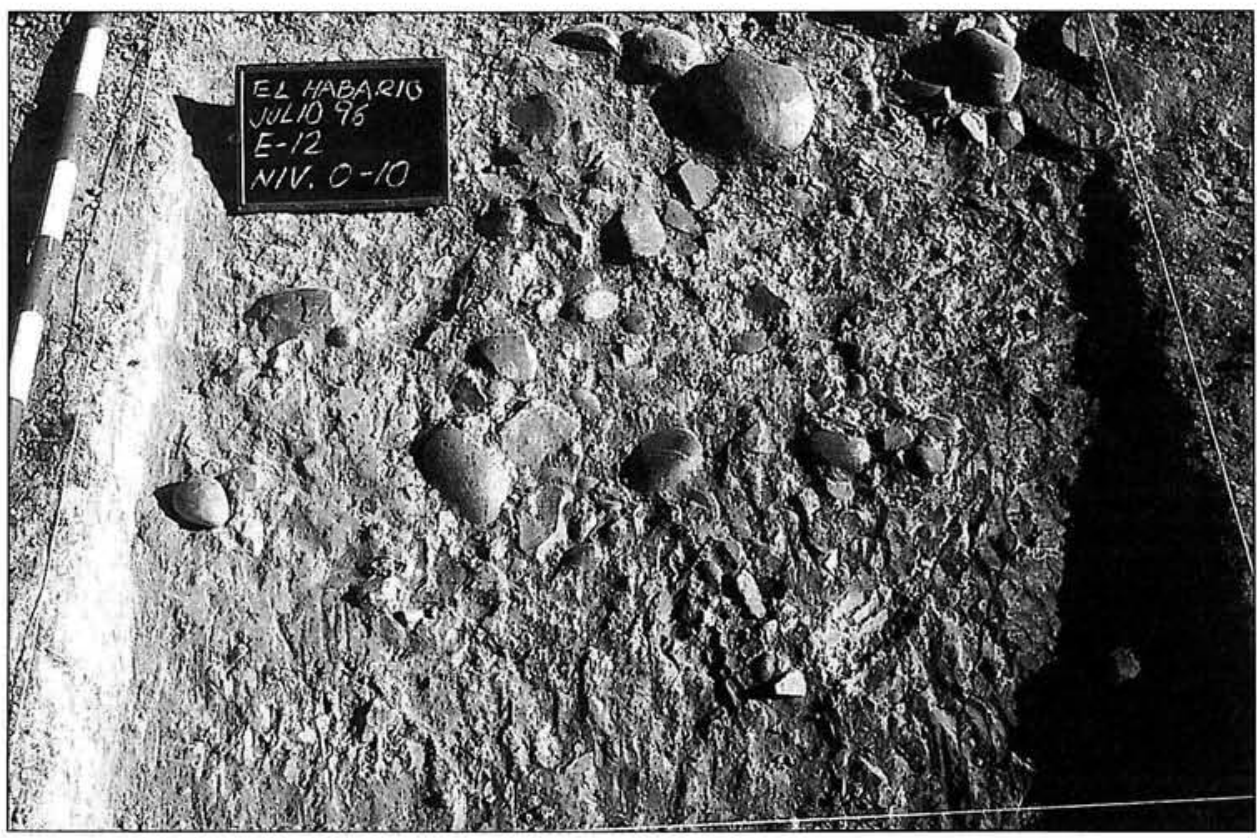

Foto 4. Detalle en cue se muestra la disposición de los materiales 


\section{BIBLIOGRAFIA}

ARLAS CABAL, P. (1987): "Estrategias de aprovechamiento de las materias primas líticas en la costa oriental de Asturias (VIII-III milenios a.C.)". MORA et al. (Eds.): Tecnologia y cadenas operativas liticas. Reunión Internacional, 15-18 Enero de 1991. pp. 37-49.

CASTANEDO, I; MUÑOZ, E., y MALPELO, B. (1993): "El yacimiento al aire libre de El Habario (Castro-Cillorigo, Cantabria)". Nivel Cero, $n .{ }^{\circ}$ 3. Santander. pp. 5-29.

GONZALEZ ECHEGARAY, J. (1957): "La Cueva de La Mora, un yacimiento paleolítico en la región de los Picos de Europa". Altamira, n. ${ }^{\circ}$ (1-3). Santander. pp. 3-26.

MuÑoz, E. et al. (1985): "Yacimientos Arqueológicos en el valle del Deva".Boletín Cäntabro de Espeleologia. n. ${ }^{\circ}$ 6. Santander, pp. 67-74.

SANTONJA, M. (1985-1985): "Los núcleos de lascas en las industrias paleolíticas de la meseta española" Zephyrus, n. ${ }^{\circ}$ XXXVII-XXXVIII. Salamanca. pp. 17-33.

TIXIER, J. (1980): Prébistoire de la pierre taillé. I. Terminologie et Technologie. París. 